\title{
Renal injury in neonates: use of "omics" for developing precision medicine in neonatology
}

\author{
Mandar S. Joshi' ${ }^{1}$ Kelsey A. Montgomery ${ }^{1}$, Peter J. Giannone' ${ }^{1}$ John A. Bauer ${ }^{1}$ and Mina H. Hanna ${ }^{1}$
}

Preterm birth is associated with increased risks of morbidity and mortality along with increased healthcare costs. Advances in medicine have enhanced survival for preterm infants but the overall incidence of major morbidities has changed very little. Abnormal renal development is an important consequence of premature birth. Acute kidney injury (AKI) in the neonatal period is multifactorial and may increase lifetime risk of chronic kidney disease.

Traditional biomarkers in newborns suffer from considerable confounders, limiting their use for early identification of AKI. There is a need to develop novel biomarkers that can identify, in real time, the evolution of renal dysfunction in an early diagnostic, monitoring and prognostic fashion. Use of "omics", particularly metabolomics, may provide valuable information regarding functional pathways underlying AKI and prediction of clinical outcomes.

The emerging knowledge generated by the application of "omics" (genomics, proteomics, metabolomics) in neonatology provides new insights that can help to identify markers of early diagnosis, disease progression, and identify new therapeutic targets. Additionally, omics will have major implications in the field of personalized healthcare in the future. Here, we will review the current knowledge of different omics technologies in neonatal-perinatal medicine including biomarker discovery, defining as yet unrecognized biologic therapeutic targets, and linking of omics to relevant standard indices and long-term outcomes.

\footnotetext{
A
} lthough improvements in clinical care have greatly increased the survival of infants born prematurely, preterm birth remains a major cause of infant mortality and morbidity. Premature infants typically have immature renal structure and function at birth and are at high risk of acute kidney injury (AKI) in the perinatal period (1). The diagnosis and management of abnormal renal function in preterm infants is confounded by several factors, including a current lack of studies testing the ability of neonatal AKI definition to predict hard clinical outcomes, heterogeneity of patient characteristics at varying gestational ages, and the limitations of traditional biomarkers (serum creatinine) to reflect injury rather than function. Therefore, it is clear that the next step in biomarker identification should focus on real time pathophysiologic events using omics technologies.

Omics refers to the collective technologies used to explore the roles, relationships, and actions of the various types of molecules that make up the phenotype of an organism. The phenotype of an organism is dynamically controlled and coordinated via a complex network of interconnected systems. Such interactions are highly adaptive and regulate the responses of the organism to external factors. Systems biology is a scientific discipline that endeavors to quantify all of the molecular elements of a biological system to assess their interactions and to integrate that information into network models. Therefore, systems biology reflects the knowledge acquired by omics in a meaningful manner (2).

From genes to metabolites, the omics technologies have progressed considerably in the medical field over the last decade secondary to the remarkable advancement in methodologies and analytical tools. In this review, we discuss the current knowledge of different omics technologies in kidney diseases, specifically in the field of neonatal nephrology.

\section{PRETERM BIRTH AND THE IMPORTANCE OF "OMICS"}

Preterm birth represents the aggregation of heterogeneous phenotypes; it is a complex disorder caused by multifactorial influences and the interplay of numerous risk factors. There is significant evidence for the importance of the intrauterine environment on the health and longevity of offspring, influenced by changes that involve aberrant gene expression caused by detrimental epigenetic markers. Both preclinical studies in mice as well as clinical studies on monozygotic twins have demonstrated that epigenetic variation can be strongly influenced by environmental factors experienced during a given lifetime (3). Several gene polymorphisms have been associated with preterm birth and/or preterm premature rupture of the membranes (pPROM) $(4,5)$ In a systematic literature review of studies that investigated genetic factors involved in both preterm delivery and $\mathrm{pPROM}$, a total of 248 single-nucleotide polymorphism (SNPs) in 102 genes were found to be significantly associated with spontaneous preterm birth with intact membranes, and 39 SNPs in 32 genes were found to be 


\section{Review | Joshietal.}

significantly associated with pPROM (6). In light of these data, understanding the "omics" in addition to the epigenetic factors is likely to play a major role in establishing the link between perinatal factors and preterm birth and may provide novel targets for intervention to prevent preterm birth.

Until recently, proteomic, genomic, and metabolomic studies were primarily utilized to characterize adult diseases. However, more recent investigations have focused on using the novel technological advances in "omics" technology to provide a better understanding of perinatal development of neonates. A critical role of placental transportation and nutrient deficiency in the pathophysiology of intrauterine growth restriction was identified through metabolomic and proteomic investigations (7). Assessment of amniotic fluid status is an established part of fetal testing mainly because it is produced by the chorion and the umbilical cord, with a significant contribution from fetal urine (8-10). The amniotic fluid composition is primarily regulated by the fetus and has been previously studied to detect certain biomarkers of kidney injury (11). Changes in the biochemistry of amniotic fluid throughout pregnancy may reflect fetal maturation. Likewise, the increase in urea and creatinine concentrations and the fall in sodium and osmolality concentration near-term have been associated with the maturation of renal function (12). Low molecular weight proteins detected in the amniotic fluid are mainly excreted by the fetus (13). These proteins are freely filtered through the fetal glomerular barrier and subsequently reabsorbed and actively metabolized within the proximal tubular cells (14).

Metabolomic profiling of amniotic fluid was able to distinguish patients who delivered at term from patients who delivered preterm. A decrease in carbohydrates was associated with preterm delivery in the presence or absence of inflammation whereas an increase in amino acid metabolites was a unique feature of preterm labor with inflammation (15). Amniotic Cystatin $\mathrm{C}$ levels are thought to be a sensitive biochemical marker for the early identification of fetuses with obstructive uropathies (12).

Additionally, using analytes from blood spots, Wilson et al. examined the associations between the degree of prematurity and the levels of amino acids, enzymes, and endocrine markers in a large cohort of infants. They concluded that children at different stages of prematurity are metabolically distinct (16). Similarly Atzori et al. found that metabolomic analysis revealed distinct urinary metabolic profiles in neonates of different gestational ages (17). The metabolomic analysis showed different urine metabolic profiles between neonates with intrauterine growth restriction and controls (18).

Given the importance of early detection of factors influencing preterm birth and related morbidities, it is critical to develop novel ways to utilize omics technologies in neonatal medicine. "Omics" can provide valuable information at many levels and there is a clear need for integration between different informative levels from genes to proteins and metabolites.

\section{AKI AND NEED FOR DEVELOPING NOVEL BIOMARKERS}

$\mathrm{AKI}$, as indicated clinically by a rise in serum creatinine ( $\mathrm{SCr}$ ) occurs in approximately $12-18 \%$ of preterm neonates (19).
Very recent studies have demonstrated that the extrauterine environment is not optimal for kidney development, and thus premature infants are at a higher risk of developing renal injury and subsequent chronic kidney disease and hypertension $(20,21)$. Current methodology identifying renal dysfunction is based upon serum creatinine and urine output (UOP). The shortcomings of SCr and UOP to define AKI are well documented including: (i) a lag between initial renal injury and creatinine rise, (ii) inability to differentiate specific site of renal injury, (iii) increasing unreliability of creatinine measurement with increasing degree of renal injury due to tubular secretion of creatinine, and (iv) renal supportive therapy, when required, removes creatinine making prognostication difficult (10). Use of SCr to diagnose AKI in neonates is of limited value since initial neonatal creatinine reflects maternal values, which decrease over time and the estimated creatinine clearance changes as growth and renal development occur. Therefore, early fast and reliable biomarkers will allow more timely diagnosis and identification of infants who may benefit from interventions targeting AKI in this vulnerable patient population.

Premature neonates are arguably at highest risk for nephrotoxicity in the newborn period given discussed risk factors. Although several novel urinary biomarkers, Kidney Injury Molecule-1 (KIM-1), Neutrophil Gelatinase-associated Lipocalin (NGAL), and N-acetyl- $\beta$-D-glucosaminidase (NAG), have shown promise as earlier indicators of both kidney injury and impaired kidney function due to nephrotoxic medications or clinical status in adults, limited data is available in neonates. As urinary biomarkers, they have demonstrated utility in evaluating kidney injury given several important features: (i) Their intrinsic origins in the kidney, (ii) established baseline values in the premature infant corresponding to gestational age, and (iii) value elevations in direct response to impaired kidney function or injury with return to baseline after the inciting event (22). Biomarkers may also have a role in identifying impaired kidney function due to nephrotoxic medications such as aminoglycosides (23). Although data quantifying nephrotoxicity related to aminoglycosides is sparse, these urinary biomarkers, specifically KIM-1, have been used to coincide with proximal tubule nephrotoxicity and resulting ramifications (24). While urinary NGAL may serve as a biomarker of sepsis-induced kidney injury, NAG's efficacy as a predictor of renal ischemic damage following perinatal asphyxia has also been demonstrated (25). Additionally, urinary NGAL and KIM-1 have been associated with AKI and long-term mortality in very-lowbirth-weight infants in single center studies $(24,26,27)$ as well as decreased function in congenital obstructive nephropathy (28). In the future, urinary biomarker utilization has the potential to identify neonatal kidney injury earlier, monitor kidney function, and follow progression. Thus, such tools could be used to improve neonatal acute and long-term outcomes.

\section{OMICS IN NEONATAL NEPHROLOGY}

\section{Neonatal Cardiac Surgery}

$\mathrm{AKI}$ is common in neonates undergoing cardiac surgery, and is associated with increased mortality and ICU length of stay (29). 
Despite significant advances in preclinical research, very few clinically relevant therapeutic options are currently available. One of the major problems is delayed diagnosis. Creatinine, which is the established biomarker of kidney injury, does not usually rise until $48 \mathrm{~h}$ postsurgery. By that time, it might be too late to intervene. Thus, there is a clear need to develop biomarkers that can enable early and reliable measurement of renal injury (30).

Novel urine protein peaks identified using a proteomics approach were associated with AKI in a prospective cohort of patients undergoing cardiopulmonary bypass (31). These peaks were subsequently identified as aprotinin, $\alpha 1$ microglobulin, $\alpha 1$-acid glycoprotein, and albumin $(32,33)$. Urinary aprotinin levels $2 \mathrm{~h}$ after initiation of cardiopulmonary bypass were predictive of AKI (92\% sensitivity, 96\% specificity, and area under the curve of 0.98) (33). Urinary $\alpha 1$ microglobulin, $\alpha 1$-acid glycoprotein and albumin were also evaluated in two prospective pediatric cardiac surgery cohorts (test set $n=30$, validation set $n=365$ ) and demonstrated an area under the curve of $0.84,0.87$, and 0.76 , respectively, at $6 \mathrm{~h}$ after cardiac surgery compared to matched controls (32). These data demonstrate that the urinary $\alpha(1)$-microglobulin, $\alpha(1)$-acid glycoprotein, and albumin may represent early biomarkers of AKI after cardiac surgery and offers prognostic information about the duration of AKI and length of hospitalization after cardiac surgery.

Mass spectrometry-based metabolomics was used in a prospective cohort of pediatric cardiac surgery patients $(n=40)$. Twenty-one of these children developed acute kidney injury as defined by an increase in serum creatinine concentrations $50 \%$ or greater from baseline after $48-72 \mathrm{~h}$. Homovanillic acid sulphate ( $\left.\mathrm{HVA}-\mathrm{SO}_{4}\right)$, a dopamine metabolite, was identified as a marker indicating AKI with $90 \%$ sensitivity and $95 \%$ specificity using a cutoff value of $24 \mathrm{ng} / \mathrm{ml}$ at $12 \mathrm{~h}$ after surgery (34).

Although these data provide promising results, there is still a need for large prospective trials to validate the usefulness of these markers in this understudied population.

\section{Drug-Induced Nephrotoxicity}

Nephrotoxic-medications are becoming increasingly recognized as a common and potentially modifiable cause of AKI in neonates. Early identification of renal injury allows the application of renoprotective approaches such as fluid administration management and adjustment of nephrotoxic medications in the early stages of AKI. One approach for the early detection of kidney injury involves defining different biomarkers that rely on the mechanisms of toxicity for each drug or drug class (35). In our experimental study, gentamicin-induced acute kidney injury in newborn rats resulted in a distinct urinary metabolic profile characterized by glucosuria, phosphaturia, and aminoaciduria that preceded changes in serum creatinine. Additionally, lower levels of kynurenic acid were noted in the urine of gentamicin injected rats, coinciding with higher levels of tryptophan, suggesting a degrading effect of gentamicin toxicity on tryptophan metabolism pathway (36). Xu et al. applied integrated pathway analysis and metabolite-transcript correlation analysis to define perturbed biochemical pathways and molecular functions that may be relevant to the mechanisms of nephrotoxicity. They concluded that transcriptional downregulation of luminal sodium-dependent transporters SLC5A1, SLC5A2, SLC6A18, and SLC16A7 might be the central mediators of drug-induced kidney injury and adaptive response pathways (37). The combined approach applied in this study demonstrates the relevance of using omics profiles to elucidate the systems toxicology.

An alternative approach involves finding a limited number of biomarkers that identify injury to primary sites in the kidney, such as the glomerulus or the proximal tubule. A recent prospective observational trial showed that aminoglycoside exposure lead to an increase in urinary biomarkers, including those that signify proximal tubular damage, irrespective of rise in $\mathrm{SCr}$ (38). We have recently utilized metabolomics technology to identify urinary biomarkers of renal injury prior to changes in serum creatinine levels (39). The lack of longitudinal studies makes it difficult to assess the long term implications of nephrotoxic induced AKI in neonates. Thus further studies in the use of omics technologies in early detection of AKI are highly valuable in order to improve short-term as well as long-term outcomes.

\section{Prematurity}

The tubular function is immature in preterm neonates; therefore, the response to abrupt homeostatic changes is suboptimal. They are also more likely to be exposed to nephrotoxic medications in early life such as gentamicin, indomethacin, and furosemide. In a single center prospective cohort, Askenazi et al. demonstrated that urinary biomarkers can predict AKI and mortality in very-low-birth-weight infants independent of gestational age and birth weight (27).

KIM-1 is a promising biomarker of proximal tubular injury associated with aminoglycosides exposure. McWilliam et al. found significant increases in three urinary biomarkers (Kidney Injury Molecule-1 (KIM-1), Neutrophil Gelatinase-associated Lipocalin (NGAL), and N-acetyl-b-D-glucosaminidase (NAG)) during treatment with multiple courses of gentamicin in a proof-of-concept study. When adjusted for potential confounders, the treatment effect of gentamicin remained significant only for KIM-1 (23). We found that urinary NGAL, osteopontin (OPN) and cystatin C (Cys C) increased significantly in infants who developed AKI. In contrast, urinary epidermal growth factor (EGF) and uromodulin (UMOD) decreased significantly in this group. Urinary biomarkers demonstrated a significant change $24 \mathrm{~h}$ prior to contemporary creatininebased neonatal AKI definition (40). Validating the association of novel biomarkers with clinically relevant hard outcomes will enlighten the expected translation into clinical practice. It is extremely essential to acknowledge the differences in omics biomarkers across different gestational ages, postnatal days, and fluid balance status when designing future validation studies (22,41-43). Additionally, recognizing centers' variations in aspects of clinical management as well as patient demographics and genetic admixture is important. 


\section{Review Joshiet al.}

\section{Obstructive Nephropathy}

Decramer et al. identified urinary biomarkers that could predict the outcome of ureteropelvic junction (UPJ) obstruction by applying urinary proteomic analysis. Using a discovery population of 13 healthy newborns, 19 UPJ obstruction patients with mild obstruction (leading to spontaneous resolution of the obstruction) and 19 UPJ obstruction patients with severe obstruction (that had to be operated on to remove the stenosis), the investigators identified a panel of 51 peptide biomarkers with differential urinary abundance between the three groups. Subsequently, these biomarkers were validated in a prospective blinded cohort of 36 new UPJ obstruction patients with intermediate UPJ obstruction. The prediction model based on the urinary peptidome was formulated before 3 mo of age and predicted the clinical outcome after 15 mo of follow-up. Using these noninvasive biomarkers, they were able to predict the clinical evolution as either surgery or spontaneous resolution of the obstruction, several months in advance and with 94\% precision (44-46). A cost-effectiveness study showed that the incorporation of urinary proteome analysis in the initial evaluation of UPJ obstruction significantly reduces costs and increases the quality-adjusted-life-year in this patient population (47). The analysis included the costs of surgery, imaging, and office visits based on hospital charge data. The incorporation of urinary proteome analysis as a marker test of disease progression was used to select out individuals with positive test as likely to progress for early surgery, and diminish the intensity of imaging and follow-up in the individuals with negative results. Using this model significantly reduced the cost and increased quality-adjusted-life-year in this patient population. In a follow-up study, the urinary proteomes of early surgically corrected UPJ obstruction patients were very similar to age-matched controls after a 5-y follow-up (48). In contrast, the urinary proteome of the conservatively followed intermediate UPJ obstruction patients, (leading either to spontaneous resolution of the obstruction or late surgical intervention), was significantly different from controls, suggesting ongoing renal or ureteral remodeling in the conservatively followed patients that was not evident clinically. Using similar approaches over the past few years, there has been a significant interest in identifying biomarkers that can assist in the accurate diagnosis of surgical cases of UPJ obstruction (49). However, there are a number of different biomarkers being studied at various centers. Hence there is a clear need for an integrated "omics" approach to develop prospective, multicenter studies to investigate the clinical value of these biomarkers.

Klein et al. studied the fetal urinary peptidome with the aim to detect biomarkers that can predict postnatal function in the prototypic congenital anomalies of the kidney and urinary tract (CAKUT) posterior urethral valves (50). Among more than 4,000 fetal urinary peptide candidates, 26 peptides were identified that were specifically associated with posterior urethral valves in 13 patients with early end-stage renal disease compared to 15 patients with absence of end-stage renal disease before the age of 2 . A classifier based on these peptides correctly predicted postnatal renal function with $88 \%$ sensitivity and $95 \%$ specificity in an independent blinded validation cohort of 38 posterior urethral valve patients. The prediction of postnatal renal function will be of high additional value for appropriate clinical follow-up such as long-term planning for implementation of renal replacement therapy. Additionally, this may be useful in providing a truly informed prenatal counseling.

\section{SUMMARY AND FUTURE APPROACHES}

The application of omics methodologies is becoming increasingly popular in pediatric/neonatology due to its unique ability to generate functional readouts of systems biology, setting the ground for future personalized prenatal, neonatal and pediatric care. Yet the clinical translation of this unprecedented source of knowledge into clinical practices for neonatal health care requires proper addressing of the inherent inter-individual variability (51). Although separate omics studies are mainly descriptive in nature, the goal is that through integration of experimental approaches and computational modelling, new insights will be generated that can help to identify marker sets to guide diagnosis, monitor postnatal metabolic maturation, and identify new therapeutic targets in a tailored manner (Figure 1). The evolving role of omics as biomarkers of renal injury will help improve earlier detection of the initial insult, and improve prediction of short term outcomes such as mortality, duration of ICU stay, and duration of mechanical ventilation. Furthermore, it may be useful to distinguish between the various types and pathogeneses of AKI (52). Characterizing the mechanisms involved in the progression of renal development and injury could have a significant impact on the outcome of affected neonates. The following stages

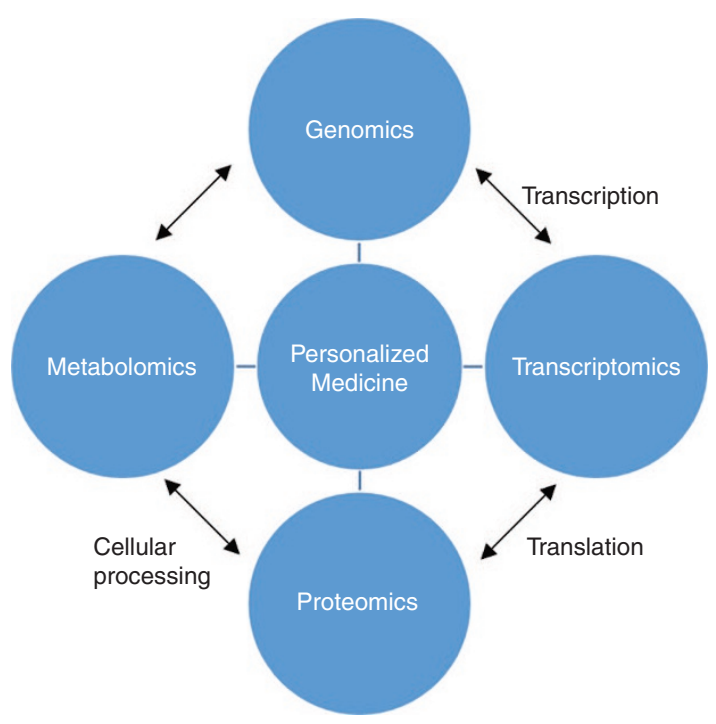

Figure 1. Evolving role of "Omics" in the development of personalized medicine. Individual "omics" studies are used to study various aspects of disease process. Integration of these various approaches will provide new insights and enable the identification of specific marker sets. Early and precise detection of these markers will allow us to develop personalized therapeutic strategies in the future. 
delineate how to translate the biomarker(s) discovery association studies into clinical applications in a stepwise approach:

1. Defining a clear clinical question: AKI vs. no AKI?

2. Selection of appropriate patient group(s), samples, and clinical data collection

3. Identification of specific biomarkers

4. Validation in a separate group of patients/samples

5. Validation on a large scale

6. Delineation of the interaction of nutrients, gene expression, and metabolism through integration of pathophysiology using pathway analysis tools.

The identification of a panel of biomarkers coming from a combination of genomics, epigenomics, transcriptomics, proteomics and metabolomics detected early will allow testing of the safety and efficacy of applying conventional or novel renoprotective approaches in response to these early-warning system markers.

\section{STATEMENT OF FINANCIAL SUPPORT}

This study was supported by Children's Miracle Network (CMN).

Disclosure: Authors have no financial relationships relevant to this article to disclose.

\section{REFERENCES}

1. Selewski DT, Charlton JR, Jetton JG, et al. Neonatal acute kidney injury. Pediatrics 2015;136:e463-73.

2. Pesce F, Pathan S, Schena FP. From -omics to personalized medicine in nephrology: integration is the key. Nephrol Dial Transplant 2013;28:24-8.

3. Kaminsky ZA, Tang T, Wang SC, et al. DNA methylation profiles in monozygotic and dizygotic twins. Nat Genet 2009;41:240-5.

4. Romero R, Espinoza J, Gotsch F, et al. The use of high-dimensional biology (genomics, transcriptomics, proteomics, and metabolomics) to understand the preterm parturition syndrome. BJOG 2006;113 Suppl 3:118-35.

5. Romero R, Velez Edwards DR, Kusanovic JP, et al. Identification of fetal and maternal single nucleotide polymorphisms in candidate genes that predispose to spontaneous preterm labor with intact membranes. Am J Obstet Gynecol 2010;202:431.e1-34.

6. Capece A, Vasieva O, Meher S, Alfirevic Z, Alfirevic A. Pathway analysis of genetic factors associated with spontaneous preterm birth and pre-labor preterm rupture of membranes. PLoS One 2014;9:e108578.

7. Cecconi D, Lonardoni F, Favretto D, et al. Changes in amniotic fluid and umbilical cord serum proteomic profiles of foetuses with intrauterine growth retardation. Electrophoresis 2011;32:3630-7.

8. Thomas DF, Gordon AC. Management of prenatally diagnosed uropathies. Arch Dis Child 1989;64(1 Spec No):58-63.

9. Seeds AE. Current concepts of amniotic fluid dynamics. Am J Obstet Gynecol 1980;138:575-86.

10. Modena AB, Fieni S. Amniotic fluid dynamics. Acta Biomed 2004;75 Suppl $1: 11-3$.

11. Jacobo-Estrada T, Cardenas-Gonzalez M, Santoyo-Sánchez M, et al. Evaluation of kidney injury biomarkers in rat amniotic fluid after gestational exposure to cadmium. J Appl Toxicol 2016;36:1183-93.

12. Mussap M, Fanos V, Pizzini C, Marcolongo A, Chiaffoni G, Plebani M. Predictive value of amniotic fluid cystatin $\mathrm{C}$ levels for the early identification of fetuses with obstructive uropathies. BJOG 2002;109:778-83.

13. Burghard R, Pallacks R, Gordjani N, Leititis JU, Hackelöer BJ, Brandis M. Microproteins in amniotic fluid as an index of changes in fetal renal function during development. Pediatr Nephrol 1987;1:574-80.

14. Scherberich JE. Urinary proteins of tubular origin: basic immunochemical and clinical aspects. Am J Nephrol 1990;10 Suppl 1:43-51.
15. Romero R, Mazaki-Tovi S, Vaisbuch E, et al. Metabolomics in premature labor: a novel approach to identify patients at risk for preterm delivery. J Matern Fetal Neonatal Med 2010;23:1344-59.

16. Wilson K, Hawken S, Ducharme R, et al. Metabolomics of prematurity: analysis of patterns of amino acids, enzymes, and endocrine markers by categories of gestational age. Pediatr Res 2014;75:367-73.

17. Atzori L, Antonucci R, Barberini L, et al. $1 \mathrm{H}$ NMR-based metabolomic analysis of urine from preterm and term neonates. Front Biosci (Elite Ed) 2011;3:1005-1012.

18. Dessì A, Atzori L, Noto A, et al. Metabolomics in newborns with intrauterine growth retardation (IUGR): urine reveals markers of metabolic syndrome. J Matern Fetal Neonatal Med 2011;24 Suppl 2:35-9.

19. Viswanathan S, Manyam B, Azhibekov T, Mhanna MJ. Risk factors associated with acute kidney injury in extremely low birth weight (ELBW) infants. Pediatr Nephrol 2012;27:303-11.

20. White SL, Perkovic V, Cass A, et al. Is low birth weight an antecedent of $\mathrm{CKD}$ in later life? A systematic review of observational studies. Am J Kidney Dis 2009;54:248-61.

21. Sutherland MR, Gubhaju L, Moore L, et al. Accelerated maturation and abnormal morphology in the preterm neonatal kidney. J Am Soc Nephrol 2011;22:1365-74.

22. Askenazi DJ, Koralkar R, Levitan EB, et al. Baseline values of candidate urine acute kidney injury biomarkers vary by gestational age in premature infants. Pediatr Res 2011;70:302-6.

23. McWilliam SJ, Antoine DJ, Sabbisetti V, et al. Mechanism-based urinary biomarkers to identify the potential for aminoglycoside-induced nephrotoxicity in premature neonates: a proof-of-concept study. PLoS One 2012;7:e43809.

24. Parravicini E, Nemerofsky SL, Michelson KA, et al. Urinary neutrophil gelatinase-associated lipocalin is a promising biomarker for late onset culture-positive sepsis in very low birth weight infants. Pediatr Res 2010;67:636-40.

25. Willis F, Summers J, Minutillo C, Hewitt I. Indices of renal tubular function in perinatal asphyxia. Arch Dis Child Fetal Neonatal Ed 1997;77:F57-60.

26. Lavery AP, Meinzen-Derr JK, Anderson E, et al. Urinary NGAL in premature infants. Pediatr Res 2008;64:423-8.

27. Askenazi DJ, Montesanti A, Hunley H, et al. Urine biomarkers predict acute kidney injury and mortality in very low birth weight infants. J Pediatr 2011;159:907-12.e1.

28. Wasilewska A, Taranta-Janusz K, Dębek W, Zoch-Zwierz W, KuroczyckaSaniutycz E. KIM-1 and NGAL: new markers of obstructive nephropathy. Pediatr Nephrol 2011;26:579-86.

29. Alabbas A, Campbell A, Skippen P, Human D, Matsell D, Mammen C. Epidemiology of cardiac surgery-associated acute kidney injury in neonates: a retrospective study. Pediatr Nephrol 2013;28:1127-34.

30. Bignami E, Landoni G, Crescenzi G, et al. Role of cardiac biomarkers (troponin I and CK-MB) as predictors of quality of life and long-term outcome after cardiac surgery. Ann Card Anaesth 2009;12:22-6.

31. Nguyen MT, Ross GF, Dent CL, Devarajan P. Early prediction of acute renal injury using urinary proteomics. Am J Nephrol 2005;25:318-26.

32. Devarajan P, Krawczeski CD, Nguyen MT, Kathman T, Wang Z, Parikh CR. Proteomic identification of early biomarkers of acute kidney injury after cardiac surgery in children. Am J Kidney Dis 2010;56:632-42.

33. Nguyen MT, Dent CL, Ross GF, et al. Urinary aprotinin as a predictor of acute kidney injury after cardiac surgery in children receiving aprotinin therapy. Pediatr Nephrol 2008;23:1317-26.

34. Beger RD, Holland RD, Sun J, et al. Metabonomics of acute kidney injury in children after cardiac surgery. Pediatr Nephrol 2008;23:977-84.

35. Bonventre JV, Vaidya VS, Schmouder R, Feig P, Dieterle F. Next-generation biomarkers for detecting kidney toxicity. Nat Biotechnol 2010;28:436-40.

36. Hanna MH, Segar JL, Teesch LM, Kasper DC, Schaefer FS, Brophy PD. Urinary metabolomic markers of aminoglycoside nephrotoxicity in newborn rats. Pediatr Res 2013;73:585-91.

37. Xu EY, Perlina A, Vu H, et al. Integrated pathway analysis of rat urine metabolic profiles and kidney transcriptomic profiles to elucidate the systems toxicology of model nephrotoxicants. Chem Res Toxicol 2008;21:1548-61. 
38. Jansen D, Peters E, Heemskerk S, et al. Tubular injury biomarkers to detect gentamicin-induced acute kidney injury in the neonatal intensive care unit. Am J Perinatol 2016;33:180-7.

39. Hanna M, Brophy PD, Giannone PJ, Joshi MS, Bauer JA, RamachandraRao S. Early urinary biomarkers of acute kidney injury in preterm infants. Pediatr Res 2016;80:218-23.

40. Hanna M, Brophy PD, Giannone PJ, Joshi MS, Bauer JA, RamachandraRao S. Early urinary biomarkers of acute kidney injury in preterm infants. Pediatr Res 2016;80:218-23.

41. Saeidi B, Koralkar R, Griffin RL, Halloran B, Ambalavanan N, Askenazi DJ. Impact of gestational age, sex, and postnatal age on urine biomarkers in premature neonates. Pediatr Nephrol 2015;30:2037-44.

42. Askenazi D, Saeidi B, Koralkar R, Ambalavanan N, Griffin RL. Acute changes in fluid status affect the incidence, associative clinical outcomes, and urine biomarker performance in premature infants with acute kidney injury. Pediatr Nephrol 2016;31:843-51.

43. Tsukahara H, Sugaya T, Hayakawa K, et al. Quantification of L-type fatty acid binding protein in the urine of preterm neonates. Early Hum Dev 2005;81:643-6.

44. Decramer S, Wittke S, Mischak H, et al. Predicting the clinical outcome of congenital unilateral ureteropelvic junction obstruction in newborn by urinary proteome analysis. Nat Med 2006;12:398-400.
45. Decramer S, Bascands JL, Schanstra JP. Non-invasive markers of ureteropelvic junction obstruction. World J Urol 2007;25:457-65.

46. Decramer S, Zürbig P, Wittke S, Mischak H, Bascands JL, Schanstra JP. Identification of urinary biomarkers by proteomics in newborns: use in obstructive nephropathy. Contrib Nephrol 2008;160:127-41.

47. Mesrobian HG. The value of newborn urinary proteome analysis in the evaluation and management of ureteropelvic junction obstruction: a costeffectiveness study. World J Urol 2009;27:379-83.

48. Bandin F, Siwy J, Breuil B, et al. Urinary proteome analysis at 5-year followup of patients with nonoperated ureteropelvic junction obstruction suggests ongoing kidney remodeling. J Urol 2012;187:1006-11.

49. Papachristou F, Pavlaki A, Printza N. Urinary and serum biomarkers in ureteropelvic junction obstruction: a systematic review. Biomarkers 2014;19:531-40.

50. Klein J, Lacroix C, Caubet C, et al. Fetal urinary peptides to predict postnatal outcome of renal disease in fetuses with posterior urethral valves (PUV). Sci Transl Med 2013;5:198ra106

51. Hanna MH, Dalla Gassa A, Mayer G, et al. The nephrologist of tomorrow: towards a kidney-omic future. Pediatr Nephrol 2016; e-pub ahead of print 9 March 2016.

52. Parikh CR, Devarajan P. New biomarkers of acute kidney injury. Crit Care Med 2008;36(4 Suppl):S159-65. 